\title{
Dermoscopy unveils the mystery of a deceptive nodule!
}

\section{Subrata Malakar ${ }^{1}$, Samipa Samir Mukherjee $^{2}$}

\author{
${ }^{1}$ Department of Dermatology, Rita Skin Foundation, Kolkata, India, ${ }^{2}$ Department of Dermatology, Cutis Academy of \\ Cutaneous Sciences, Bangalore, India
}

Corresponding author: Dr. Samipa Samir Mukherjee, E-mail: drsamipamukherjee@gmail.com

Sir,

Dermoscope is an office based non invasive diagnostic tool based on the principle of compilation of a magnifying component with a light source. Polarized dermoscopy enables examination of the reflection of light shone over the epidermal surface without actual physical contact. Besides the routine use of this tool in the diagnosis of dermatological disorders, they also provide a wider range of applications for the practitioner. Reports state that up to $38 \%$ of soft tissue foreign bodies (STFBs) are neglected in initial clinical examinations in emergency departments, and $25 \%$ of all STFBs are presented weeks, months, and even years after a penetrating injury $[1,2]$. The limited size of broken particles, thorns, wood splinters or glass splinters make it extremely difficult to detect in the skin. Only magnification can reassure that a disturbing skin lesion is free of a foreign substance which can lead to a delay in the healing process. We herein describe a case of persistent nodule for last four years diagnosed as retained suture material granuloma through dermoscopy.

A 45 year old farmer, with the history of walking barefoot presented to the outpatient department with a minimally tender inflamed nodule over the dorsum of the right foot of 4 years duration. History revealed on and off swelling of the lesion with one episode of pus discharge about 4 years back for which surgical intervention was done at a local hospital. Although the discharge resolved post the intervention the wound did not seem to reduce and went on to slowly increase in size and become a hyperpigmented firm nodule with minimal tenderness and inflammation at present (Fig. 1). A biopsy of the lesion was planned keeping in mind the possibility of a granulomatous origin.

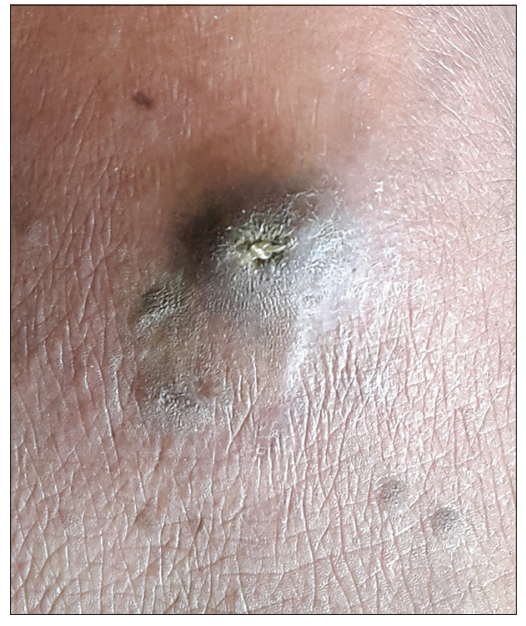

Figure 1: Solitary hyperpigmented nodule over dorsum of foot.

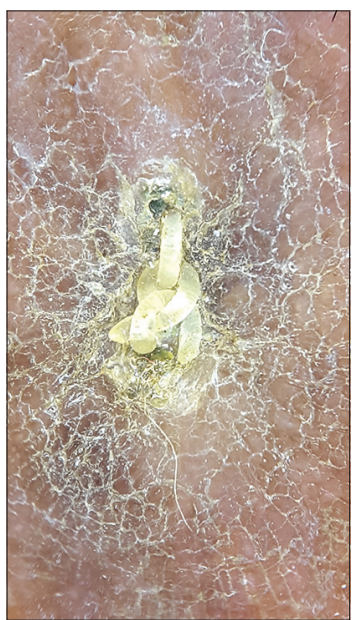

Figure 2: Dermatoscopy showing suture knot in the centre of the lesion.

However as a routine diagnostic procedure dermoscopy of the lesion was done which revealed a knot leading to the confirmation of diagnosis of a retained suture material granuloma (Fig. 2). The patient was further managed with removal of the retained suture and topical corticosteroids to reduce local inflammation.

\footnotetext{
How to cite this article: Malakar S, Mukherjee SS. Dermoscopy unveils the mystery of a deceptive nodule!. Our Dermatol Online. $2017 ; 8(4): 495-496$. Submission: 01.12.2016; Acceptance: 06.04.2017

DOI: 10.7241 /ourd.20174.141
} 
The likelihood of STFB oversight depends upon the presence of clinical signs like tenderness, swelling, and hematoma following injury as well as on the nature, size, location, and number of foreign bodies. Retained foreign bodies may be the result of post traumatic incident, negligence by patient or iatrogenic. The limited size of broken particles, thorns, tiny gravel pieces, wood splinters or glass splinters make it extremely difficult to detect in the skin. Only magnification can reassure that a persistent skin lesion is free of a foreign substance which can lead to a delay in the healing process. Although there has been a report on dermoscopic prevention of retained sutures [3], to the best of our knowledge this is the first case report from India where the retained foreign body was detected on dermoscopy thus avoiding the need for biopsy. We reinforce the use of dermoscope in routine clinical practice especially in resource poor areas where the need for biopsy may thus be obviated.

\section{REFERENCES}

1. Anderson MA, Newmeyer WL $3^{\text {rd }}$, Kilgore ES Jr. Diagnosis and treatment of retained foreign bodies in the hand. Am J Surg. 1982;144:63-7.

2. Steele M, Tran L, Watson W, Muelleman RL. Retained glass foreign bodies in wounds: Predictive value of wound characteristics, patient perception, and wound exploration. Am J Emerg Med. 1998;16:627-30.

3. Naimer SA. Dermoscopic prevention and improved detection of retained sutures. J Am Acad Dermatol. 2014;70:e57-8.

Copyright by Subrata Malakar, et al. This is an open-access article distributed under the terms of the Creative Commons Attribution License, which permits unrestricted use, distribution, and reproduction in any medium, provided the original author and source are credited. Source of Support: Nil, Conflict of Interest: None declared. 